\title{
CONTROL PROBLEM CLASSIFICATION FOR A PLASMA PROCESS
}

\author{
Petar Iordanov*,** John V. Ringwood* \\ Seán Doherty* \\ * Department of Electronic Engineering, NUI Maynooth, \\ Maynooth, IRELAND \\ **Email: petar.iordanov@eeng.nuim.ie, \\ Tel.: +353-1-7086382, Fax: +353-1-7086027
}

\begin{abstract}
The use of first-principles based models of plasma processes is discussed in this paper. Such processes are essentially highly nonlinear, feature complex interactions and are difficult to analyse. As an illustration of the nature of firstprinciple based models, a characterisation of a simple plasma process is presented in this work. Quantification of the nonlinearity, in terms of steady-state and dynamics behavior, is carried out for the studied process. The use of Hammerstein model and its applicability to the plasma process is also investigated. A basic stabilising controller for the simple plasma process is designed and its performance is analysed. Copyright (C)2005 IFAC
\end{abstract}

Keywords: physical models, process control, nonlinearity, linearization

\section{INTRODUCTION}

Electronics manufacturing is an emerging area for application of control technology. Over the last few years it has been widely recognised that a large fraction of semiconductor processing is carried out in an essentially open loop manner. Etching is, probably, the most fundamental of the semiconductor manufacturing processes. In recent years, the reactive ion etching (RIE) process has received tremendous attention and has practically become without an alternative in the semiconductor industry. RIE is a low pressure plasma system which involves chemical and physical reactions to achieve an etching process with suitable properties. Etching is a quite demanding process, and properties such as selectivity, uniformity and anisotropy are of great importance, (Badgwell et $a l ., 1995)$. It should be noted that a relatively small percentage of the chips that are produced through plasma etching are usable. At present, the decrease in device feature size, and the in- crease in aspect ratio and wafer size, have made it increasingly difficult to achieve a flawless etch. The lack of on-line sensing and closed-loop control can result in a large volume of defective materials that are manufactured before they are detected. This is why the control of plasma processes is a challenge that has inspired many researchers, in the recent years.

Until recently, control of semiconductor etching has typically been run-to-run and based on exsitu measurements of the etched wafers, (Rosen et al., 2002). Recent developments of various in-situ sensors including spectroscopic ellipsometry, has now made it possible to consider the etch control in real-time. Due to many diverse difficulties (such as influence of chamber geometry, accuracy of measurement sensors, etc.), the feedback realtime control of plasma process has become an appealing challenge. It should be noted, however, that the process control largely depends on the modelling itself. The modelling stage is the one 
that requires a thorough understanding of the plasma dynamics, which is quite often hard to analyse. The physical and chemical mechanisms in RIE are known to be very complex, and are currently not completely understood.

While a lot of work has been done on firstprinciples based models for RIE, various literature sources have pointed out that these models are not suitable for control system design and analysis, (Vincent et al., 1994). In practice, plasma models are exclusively obtained through system identification, thus ignoring any physical relationships inherent in the process that may be known. Neural network based models and linear/Hammerstein models are quite widely used in etching, (Kim and Park, 2002; Vincent et al., 1994; Erten et al., 1996; Stokes and May, 2000; VanAntwerp et al., 1997). A major drawback of the linear models is that they are normally only adequate for a relatively small region about the operating point. Artificial intelligence based models (such as fuzzylogic and neural network models) generally do not allow full understanding of the inherent process dynamics. System identification based control methodologies are often exploited to ensure process stability and disturbance rejection and to satisfy some performance criteria, for a particular plasma reactor.

The lack of much literature dealing with the application of first-principle based models for feedback control has led to a number of questions that need to be addressed. In particular, these questions concern the complexity and adequacy of such models as well as amenability to analysis and feedback control. The application of first-principle based models of plasma processes is studied in this paper, as an attempt to answer some of the questions. As an illustrative example, a model of a simple plasma process is presented in Section 2 , and its charactersitics are examined in Section 3. A controller design for the underlying plasma process is considered in Section 4, and finally, conclusions are drawn in Section 5.

\section{PLASMA PROCESS MODEL}

Description of a global model of a simple plasma process is now presented. Here, global means that spatial gradients within the chamber are not considered, so that the concentration of each chemical species inside the chamber is described by a single state variable. The process itself is a one-species plasma confined in a cylindrical chamber. The model is based on known physical interactions that are present in the plasma. Four nonlinear differential equations describe the dynamics of the process, (Turner, 2003; Iordanov, 2004):

$$
\begin{aligned}
& \frac{d n}{d t}= k_{i} N n-\frac{A}{V} \Gamma_{n} \\
& \frac{d N}{d t}=\frac{F}{M V}-\frac{A_{E} \Gamma_{N}}{V}-k_{i} N n+\frac{\left(A-A_{E}\right) \Gamma_{n}}{V} \\
& \frac{d}{d t}\left(\frac{3}{2} n k_{B} T_{e}\right)=\frac{P}{V}-\epsilon_{i} k_{i} N n-\epsilon_{*} k_{*} N n- \\
& \quad \frac{3}{2} \delta\left(k_{B} T_{e}-k_{B} T_{g}\right) k_{e} N n- \\
& \quad \frac{A}{V}\left[\frac{5}{2}+\frac{1}{2} \ln \left(\frac{M}{2 \pi m}\right)\right] k_{B} T_{e} \Gamma_{n} \\
& \frac{d}{d t}\left(\frac{3}{2} N k_{B} T_{g}\right)=\frac{3}{2} \frac{F}{M V} k_{B} T_{i n}-\frac{3}{2} k_{B} T_{g} \frac{A_{E}}{V} \Gamma_{N}+ \\
& \quad \frac{3}{2} \delta\left(k_{B} T_{e}-k_{B} T_{g}\right) k_{e} N n+ \\
& \quad \frac{3}{2} \gamma \frac{\left(A-A_{E}\right)}{V}\left(k_{B} T_{w}-k_{B} T_{g}\right) \Gamma_{N}
\end{aligned}
$$

Equations 1-4 are, respectively, balance equation for charged particles, balance equation for neutral density, electron temperature balance equation and gas temperature balance equation. The four variables, which will further be referred to as state variables, are:

\begin{tabular}{cll}
\hline$n$ & - & electron (plasma) density \\
$N$ & - & neutral density \\
$T_{e}$ & - & electron temperature \\
$T_{g}$ & - & temperature of other gas species \\
\hline
\end{tabular}

Here, the controlled variable is the ion flux, $\Gamma_{n}$. Control can be established through the following manipulated variables:

\begin{tabular}{rll}
\hline$F$ & - gas flow rate \\
$P$ & - & RF power \\
$A_{E}$ & - exhaust port area \\
$T_{w}$ & - temperature of chamber wall \\
$T_{i n}$ & - temperature of inlet gas \\
\hline
\end{tabular}

Since the latter two variables, $T_{w}$ and $T_{i n}$, have little effect on the ion flux, they are eliminated as manipulated variables. A list of the other parameters involved in equations $1-4$ is given in table 1 . Note that $k_{i}=f\left(T_{e}\right)$ and $k_{*}=f\left(T_{e}\right)$. The ion flux, $\Gamma_{n}$, and the neutral flux, $\Gamma_{N}$, are nonlinear functions of the state variables $n, N, T_{e}$ and $T_{g}$. Typical expressions for $\Gamma_{n}$ and $\Gamma_{N}$ are

$$
\begin{gathered}
\Gamma_{n}=n h \sqrt{\frac{k_{B} T_{e}}{M}} \\
\Gamma_{N}=\frac{N}{4} \sqrt{\frac{8 k_{B} T_{g}}{\pi M}}
\end{gathered}
$$

\section{PROCESS CHARACTERISATION}

\subsection{Model Adequacy}

An important question that may arise is to what extent the considered model is realistic. It should 
be stressed that a typical RIE process involves at least ten chemical species. In this study, several assumptions related to the description of the current plasma model are considered, (Turner, 2003). Firstly, the approximation that all species other than electrons have the same temperature is justified if there are sufficiently frequent collisions that share energy between particles of different species. Secondly, it is assumed that there are no losses in delivering RF power to the chamber. In addition, no terms that account for the dynamics of the actuators are present. However, the question of greater interest here is not whether this model is sufficiently representative, but can such a process description be of use from a control perspective.

\subsection{Model Characteristics}

The extent to which process nonlinearities may be significant (for an explicitly stated task) is often assessed through experience and general rules of thumb. A notable feature of the plasma model under consideration is the presence of $\mathrm{Ar}$ rhenius type of nonlinearities (terms like $e^{-E / R T}$ ). Such nonlinearities are often classified as "severe", while polynomial nonlinearities are described as "mild", (Nikolaou and Hanagandi, 1995). The location of equilibrium points of models with severe nonlinearities (as the plasma model considered in this paper) can be a formidable task. Specifically, to derive analytical solutions for the steady-states by using equations $1-4$, is a significantly timeconsuming problem. Numerical differentiation is an alternative approach to find the equilibria, although not fully reliable. This approach can be numerically sensitive, in particular, if the system of differential equations is stiff. In other words, if the model exhibits very fast and very slow time constants, this would inevitably affect the numerical differentiation. A notable feature of the

Table 1. Plasma model parameters

\begin{tabular}{|ll|}
\hline Par. & Description \\
\hline$A$ & Internal surface area of the chamber \\
$h$ & Volume of the chamber \\
& Parameter that accounts for \\
$M$ & reduction on $n$ near the walls \\
$m$ & Neutral mass \\
$\delta$ & Electron mass \\
$\epsilon_{i}$ & Mass ratio, $2 m / M$ \\
$\epsilon_{*}$ & "Nonisation energy \\
$k_{B}$ & Boltzman constant \\
$k_{e}$ & Energy rate \\
$k_{i}$ & Ionisation rate, $k_{i}=k_{i}^{(0)} e^{-\left(\frac{\epsilon_{i}}{k_{B} T_{e}}\right)}$ \\
$k_{*}$ & "Non-Ionisation" rate, $k_{*}=k_{*}^{(0)} e^{-\left(\frac{\epsilon_{*}}{k_{B} T_{e}}\right)}$ \\
$\gamma$ & Accommodation coefficient, $\gamma \in[0,1]$ \\
\hline
\end{tabular}

system of differential equations 1-4 is their stiff nature. For the considered model, at certain operating points the ratio of the largest and smallest time constants can be as large as $10^{5}$. It should be noted that at each operating point, defined by $\left\{F^{i}, P^{i}, A_{E}^{i}\right\}$, only one plausible steady state solution, $\left\{n^{\circ}, N^{\circ}, T_{e}^{\circ}, T_{g}^{\circ}\right\}$, can be found.

\subsection{Quantification of Nonlinearity}

Two aspects of the nonlinearity are addressed in this study. The first one considers the deviation from linearity at DC (static response), while the second pays attention to the transient behavior (dynamic response).

There are various ways to quantify the degree of nonlinearity in terms of DC input-output (I/O) response. The I/O relationship of $\Gamma_{n}$ as a function of each manipulated variable, $F, P$ and $A_{E}$, is illustrated in figures 1,2 and 3 , respectively. Here, $F \in\left[10^{-5}, 10^{-3}\right] \mathrm{kg} / \mathrm{s}, P \in[400,1200] \mathrm{W}$, $A_{E} \in\left[10^{-5}, 10^{-2}\right] \mathrm{m}^{2}$. It can be seen from the figures that both functions $\Gamma_{n}(F)$ and $\Gamma_{n}\left(A_{E}\right)$ are notably nonlinear, in particular, for small argument values. It is interesting to note that $\Gamma_{n}$ is visibly linear in the RF power, $P$. In this study, the degree of nonlinearity at a specified operating point $u^{x}=\left\{F^{x}, P^{x}, A_{E}^{x}\right\}$ and an interval $\delta u^{x}$ around that point, is defined by the following expression

$$
\rho\left(u^{x}, \delta u^{x}\right)=\max \left|\frac{\Gamma_{n}^{l}(u)-\Gamma_{n}(u)}{\Gamma_{n}^{l}(u)}\right| \times 100 \%
$$

where $u \in\left[u^{x}-\delta u^{x}, u^{x}+\delta u^{x}\right]$. In this equation, $\Gamma_{n}($.$) is the output of the nonlinear model, while$ $\Gamma_{n}^{l}($.$) is the tangent constructed at the operating$ point $u^{x}$ and represents the linearised I/O static map. It can be seen from its definition that $\rho($.) measures the deviation from linearity at the edges of the operating range under consideration. Essentially, this metric can be used to select operating intervals for which linearisation is reasonable. For instance, if a reasonable criterion for linearisation is $\rho(.) \leq 2.5 \%$, then an appealing objective is to maximise $\delta u^{x}$ over $u$ so that this inequality is satisfied. For this plasma model, the operating region that best fits these requirements is given by

$$
\begin{aligned}
u^{x} & =\left\{8 \times 10^{-4} \mathrm{~kg} / \mathrm{s}, 850 \mathrm{~W}, 8 \times 10^{-3} \mathrm{~m}^{2}\right\} \\
\delta u^{x} & =\left\{ \pm 2 \times 10^{-4} \mathrm{~kg} / \mathrm{s}, \pm 350 \mathrm{~W}, \pm 2 \times 10^{-3} \mathrm{~m}^{2}\right\}
\end{aligned}
$$

Clearly, this means that in the worst case, and within this operating range, the true (nonlinear) gain differs not more than $2.5 \%$ from its linear approximation.

Needless to say, a single linear model cannot be used to adequately represent the nonlinear process 
over the operating space. However, quite often a Hammerstein-type model is considered in cases when at steady-state the controlled variable(s) depends nonlinearly on the manipulated variable(s). The Hammerstein model, shown in figure 4, belongs to the group of block-oriented models, defined by cascade and/or parallel connection of static nonlinearities and linear dynamics. Because of its relatively simple structure, this model has become increasingly popular, in particular, for the modelling of chemical processes. This model combines a linear dynamics with nonlinear steadystate gain. Note that the Hammerstein model structure can be justified if the process dynamics are consistent over the entire operating range.

Nonlinearity not only affects the DC gain, but more importantly, the process dynamics is often dependent on the operating point. In other words, the time constants $\tau_{i}$ (and possibly overshoot and damping) can depend on the steadystate reference value, $u^{x}$, and the instantaneous deviation of the input from this reference value, $\delta u^{x}$. To analyse the dynamics of the presented plasma model, step input changes were applied at a grid of different operating points spanning the operating space. Step changes in one input at a time were applied and the size of the step was $10 \%$ of the steady-state reference value. For this analysis, step responses of the model state variables were considered. In this investigation, it was found that the process dynamics strongly depend on the manipulated control variable $A_{E}$, and to a less extent on $F$. As $A_{E}$ increases from $10^{-5}$ to $10^{-2}$, the time-constants $\tau_{i}$ decrease from roughly $4 \mathrm{~s}$ to about $4 \mathrm{~ms}$. It should be pointed out that even for a fixed $A_{E}$, the variation of timeconstants and overshoot due to step changes in $F$ and $P$, is still considerable. Variations in timeconstants $(\tau)$ and overshoot $(\sigma)$ were identified with the help of the following two metrics:

$$
\begin{aligned}
\delta \tau & :=\frac{\tau_{\max }-\tau_{\min }}{\tau_{\max }+\tau_{\min }} \\
\delta \sigma & :=\frac{\sigma_{\max }-\sigma_{\min }}{\sigma_{\max }+\sigma_{\min }}
\end{aligned}
$$

Essentially, equations 8 and 9 are expressions for the uncertainty in $\tau$ and $\sigma$. For a particular operating range, both parameters can be written as

$$
\begin{gathered}
\tau \in\left[\tau^{\circ}(1-\delta \tau), \tau^{\circ}(1+\delta \tau)\right] \\
\sigma \in\left[\sigma^{\circ}(1-\delta \sigma), \sigma^{\circ}(1+\delta \sigma)\right]
\end{gathered}
$$

where $\tau^{\circ}$ and $\sigma^{\circ}$ are the nominal (mean) values. An interesting fact is that $\delta \tau$ is inversely proportional to $F$. Note that it is possible to achieve consistency in time-constants and overshoot within some appropriately selected operating regions. In the following operating range

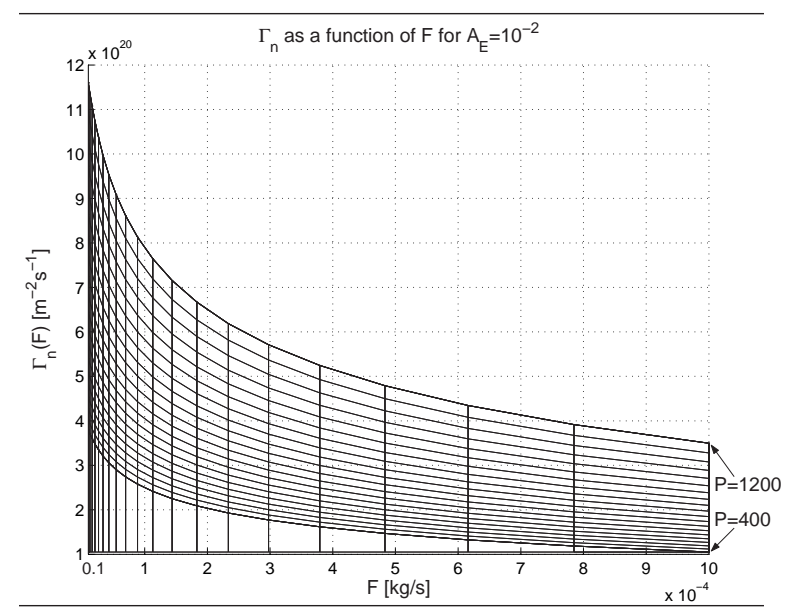

Fig. 1. Steady-state response of $\Gamma_{n}(F)$

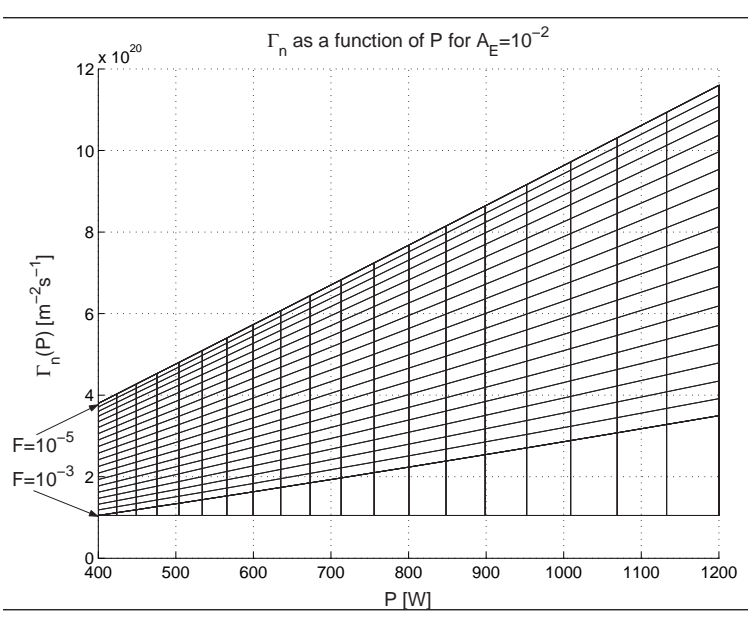

Fig. 2. Steady-state response of $\Gamma_{n}(P)$

$$
\begin{aligned}
& F \in\left[10^{-4}, 9 \times 10^{-4}\right], \\
& P \in[500,1200], A_{E}=10^{-5}
\end{aligned}
$$

process parameter variations were reasonable $(\delta \tau<25 \%$ and $\delta \sigma<0.5 \%)$, which suggests that local linearisation can be performed. Although a Hammerstein model cannot fully represent the nonlinear dynamics over the entire operating range, for the operating range in equation 12 such a model structure can be exploited.

An appealing question that may arise is how to separate the static nonlinearity from the linear dynamics part without sacrificing the physical parameters that naturally describe the process. The process interactions represented by these parameters help us to understand their effect on various model properties, such as stability, controllability, observability, numerical sensitivity, etc. Ongoing research is focusing on the development of more sophisticated, "robust" models, which will be able to describe various plasma processes by simply adjusting the relevant parameters accordingly. 


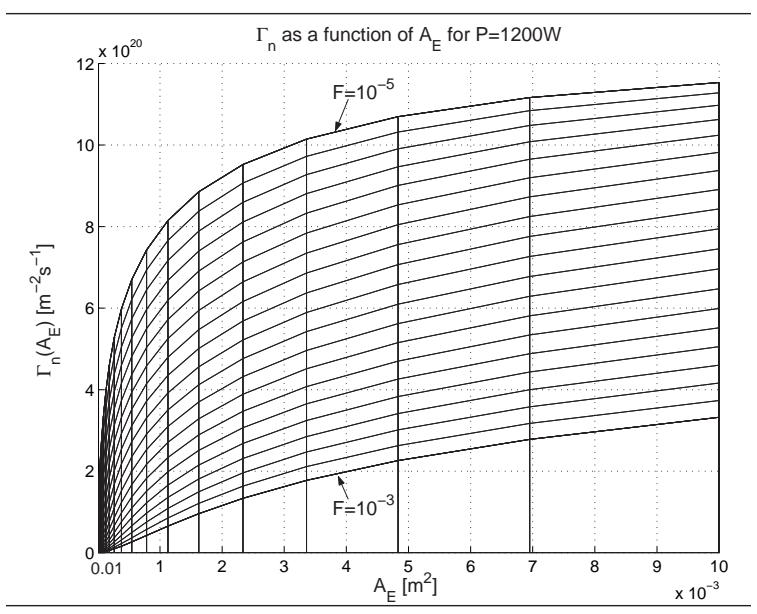

Fig. 3. Steady-state response of $\Gamma_{n}\left(A_{E}\right)$

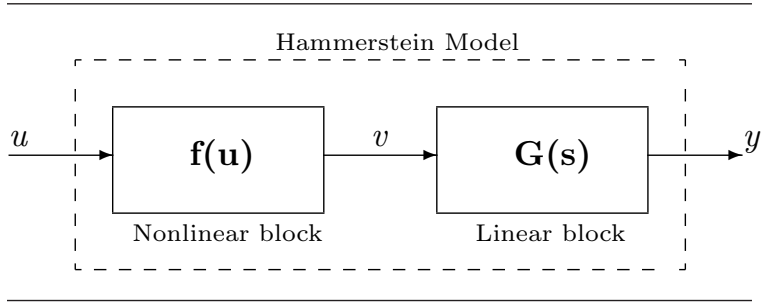

Fig. 4. Hammerstein Model Block Diagram

\subsection{Jacobian Linearisation}

The nonlinear plasma model represented by equations 1-4 was linearised using Taylor series expansion, and closed-form expressions for the statespace matrices were obtained. The linear statespace model is described by

$$
\begin{aligned}
& \dot{\mathbf{x}}=\mathbf{A x}+\mathbf{B u} \\
& \mathbf{y}=\mathbf{C x}
\end{aligned}
$$

where

$$
\mathbf{x}=\left[n, N, T_{e}, T_{g}\right]^{T}, \quad \mathbf{u}=\left[F, P, A_{E}\right]^{T}, \quad y=\Gamma_{n}
$$

All of the state-space matrices: $\mathbf{A}, \mathbf{B}$ and $\mathbf{C}$ are functions of $\mathbf{u}$, in an explicit or implicit way. An interesting fact is that the state matrix, $\mathbf{A}$, is ill-conditioned regardless of the operating point about which linearisation is performed. Here, the numerical sensitivity of the state matrix is assessed with the help of a singular value decomposition. The sensitivity analysis has shown that the first two singular values, $\sigma_{1}(\mathbf{A})$ and $\sigma_{2}(\mathbf{A})$, are significantly larger than the other two, $\sigma_{3}(\mathbf{A})$ and $\sigma_{4}(\mathbf{A})$. As a consequence, the condition number of $\mathbf{A}$, defined as $\sigma_{1}(\mathbf{A}) / \sigma_{4}(\mathbf{A})$, is of the order of $10^{30}$. Since the state matrix is poorly conditioned, any time-domain analysis will possibly deliver misleading results that are difficult to evaluate. It should be noted that for the considered operating space, all eigenvalues are real and negative, which indicates that the system is stable. The eigenvalues and singular values of the state matrix derived at the operating point $\left\{F=10^{-3}, P=\right.$ $\left.1000, A_{E}=10^{-5}\right\}$, are shown in table 2 .
Table 2. Some typical eigenvalues and singular values of $\mathbf{A}$

\begin{tabular}{|ll|}
\hline Eigenvalues of $\mathbf{A}$ & Singular Values of $\mathbf{A}$ \\
\hline$\lambda_{1}=-3.77276 \times 10^{7}$ & $\sigma_{1}=5.92859 \times 10^{21}$ \\
$\lambda_{2}=-9.56162 \times 10^{2}$ & $\sigma_{2}=8.68627 \times 10^{15}$ \\
$\lambda_{3}=-4.62219 \times 10^{2}$ & $\sigma_{3}=3.96439 \times 10^{2}$ \\
$\lambda_{4}=-3.65322 \times 10^{-1}$ & $\sigma_{4}=2.08531 \times 10^{-14}$ \\
\hline
\end{tabular}

Overcoming the numerical sensitivity of the linear state-space representation is normally addressed by regularisation/normalisation techniques. Such techniques offer reduction on the condition number of $\mathbf{A}$, and are generally based on similarity transformations. Although a desired numerical stability can often be achieved in practice, however, similarity transformations typically induce fictitious states that have no explicit relation to the original physical states. The Balanced realisation technique, (Moore, 1981), is a widely used regularisation approach. The use of this technique was considered in the present study. Although this method normally works well in practice, it was not the case with the studied linear statespace model. It is worthy to mention that the balanced regularisation technique failed to reduce the condition number of the state matrix at some operating ranges, and generally, its effect was insignificant. This perhaps can be explained with the very large condition number of $\mathbf{A}$, which seems to be a burden for any existing regularisation technique.

Analysis results have indicated that the controllability matrix is of rank two, which clearly underlines that the process model is uncontrollable. The states $T_{e}$ and $T_{g}$ are linear combinations of the states $n$ and $N$, thus indicating that the system is, however, physically controllable.

\section{SUBSYSTEM CONTROLLER DESIGN}

In order to illustrate the applicability of the studied model for feedback control, a simple controller was synthesised. For this design, a command tracking problem is considered with $\Gamma_{n}$ as controlled variable, and $F$ and $P$ as manipulated variables. Due to the aforementioned numerical difficulties, the original linearised state-space model is practically inappropriate for this purpose. Using Gramian-based balancing, the linear state-space model was transformed into a more numerically stable form and model reduction was applied. As a result, the two states corresponding to significantly faster dynamics were truncated. The linear model was obtained at the operating point $\left\{F=8 \times 10^{-4}, P=850, A_{E}=8 \times 10^{-3}\right\}$, which is consistent with the studied plasma chamber.

The considered control design is a mixed-sensitivity $\mathcal{H}_{\infty}$ problem. The need of robust control method- 


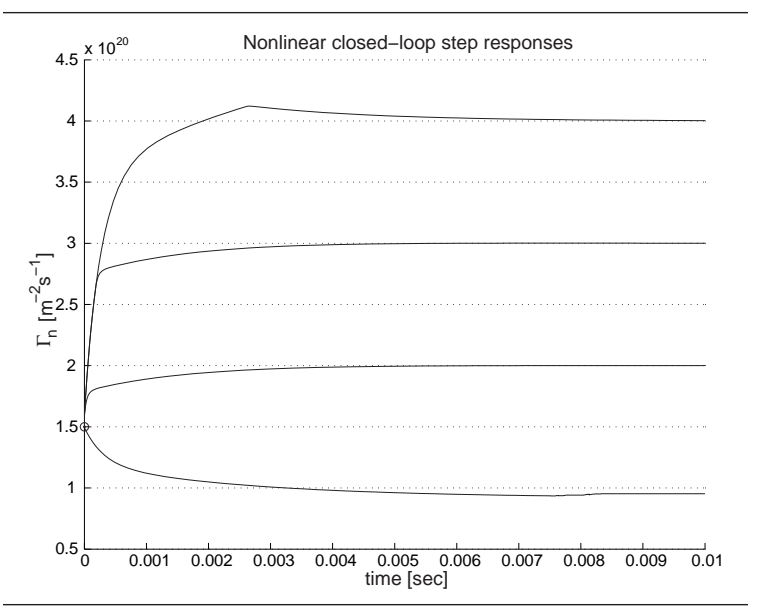

Fig. 5. Step responses of $\Gamma_{n}$

ology is justified by the eliminated high-frequency dynamics, a result of the model order reduction, and the existing dependence on the operating conditions. The design objective is expressed by:

$$
\gamma=\min _{K}\left\|\left[\begin{array}{l}
W_{1} S \\
W_{2} K S \\
W_{3} T
\end{array}\right]\right\|_{\infty}
$$

where $K$ denotes the controller, $S$ is the sensitivity function and $T$ is the complementary sensitivity function, (Skogestad and Postlethwaite, 1996). The weighting filters $W_{1}, W_{2}$ and $W_{3}$ are selected so that the closed-loop maintains stability against unstructured uncertainty and also provides good tracking performance. A controller $K$ ensuring robust performance of $\gamma=0.108$ was produced with the following choice of filters:

$$
\begin{aligned}
& W_{1}(s)=\frac{1100}{50 s+1}, \quad W_{2}=\left[\begin{array}{ll}
10^{6} & 10^{4}
\end{array}\right] \\
& W_{3}(s)=\frac{10^{-7} s+3 \times 10^{-6}}{10^{7} s+1}
\end{aligned}
$$

Closed-loop step responses, for the nonlinear plant, illustrating the command tracking of four different reference values for $\Gamma_{n}\left(1 \times 10^{20}, 2 \times 10^{20}\right.$, $3 \times 10^{20}, 4 \times 10^{20}$ ) are depicted in figure 5 . These values cover the operating space in equation 12 .

\section{CONCLUSIONS}

In this paper, analysis and feedback design of a simple plasma process have been considered, featuring a first-principle model as a basis for control design. The foregoing analysis reveals that such models are typically quite complex, highly nonlinear and their analysis highlights various numerical difficulties that are present. However, the consideration of physically-based models for design is an appealing challenge that should not be disregarded. A robust control design following model order reduction is shown to be able to achieve nominal performance across a typical operating range.

\section{ACKNOWLEDGEMENTS}

This work has been undertaken within the PlasMAC project, a joint collaboration between National University of Ireland at Maynooth and Dublin City University, and supported by Science Foundation Ireland (SFI).

\section{REFERENCES}

Badgwell, T.A., T. Breeduk, S.G. Bushman, S.W. Butler, S. Chatterjee, T.F. Edgar, A.J. Toprac and I. Trachtenberg (1995). Modelling and control of microelectronics material processing. Computers Chemical Engineering 19, 1-41.

Erten, G., A. Gharbi, F. Salam, T. Grotjohn and J. Asmussen (1996). Using neural networks to control the process of plasma etching and deposition. In: IEEE Proc.. pp. 1091-1096.

Iordanov, P. (2004). Modelling and analysis of a simple plasma process, technical report EE/JVR/4/04, NUI Maynooth, Ireland.

Kim, B. and S. Park (2002). Characterization of inductively coupled plasma using neural networks. IEEE Transactions on Plasma Science 30(2), 698-705.

Moore, B. (1981). Principal component analysis in linear systems: controllability, observability and model reduction. In: IEEE Transactions on Automatic Control. Vol. 26. pp. 17-32.

Nikolaou, M. and V. Hanagandi (1995). Nonlinearity quantification and its application to nonlinear system identification. Chemical Engineering Communications.

Rosen, I.G., T. Parent, R. Mancera, P. Chen and A. Madhukar (2002). Modelling and simulation of thermal chlorine etching of gallium arsenide with application to real-time feedback control. Mathematical and Computer Modelling 35, 335-360.

Skogestad, S. and I. Postlethwaite (1996). Multivariable Feedback Control : Analysis and Design. Wiley. London.

Stokes, D. and G.S. May (2000). Real-time control of reactive ion etching using neural networks. In: IEEE Transactions on Semicnductor Manufacturing. Vol. 13. pp. 469-480.

Turner, M. (2003). DCU simple plasma model, private communication.

VanAntwerp, J., R. Braatz and R. Sahinidis (1997). Robust nonlinear control of plasma etching. Electrochemical proceedings 97(10), 454-462.

Vincent, T.L., P.P. Khargonekar, B.A. Rashap, F. Terry and M. Alta (1994). Nonlinear system identification and control of a reactive ion etcher. In: Proceedings of the American Control Conference. Baltimore, Maryland. pp. 902-906. 